- Original Article

\title{
The Relationship between Heart Rate Variability and Aortic Knob Width
}

\author{
Si-Yeun Sung, Jee-Hye Han*, Jung-Hwan Kim, Kil-Young Kwon, Seong-Wook Park \\ Department of Family Medicine, Nowon Eulji Medical Center, Eulji University, Seoul, Korea
}

Background: The aortic knob width (AKW) and the heart rate variability (HRV) were suggested to be related to development of cardiovascular diseases. However, the relationship between them has not been investigated; thus, this study aimed to determine this relationship.

Methods: This study included 587 Koreans aged 18-79 years. Their physical measurements, medical and social histories, blood test findings, and chest radiographs were obtained. The HRV parameters included the standard deviation of the N-N interval (SDNN), root mean square of successive differences (RMSSD), approximate entropy (ApEn), total power (TP), very low frequency (VLF), low frequency (LF), high frequency (HF), and LF/HF ratio, which were measured for 5 minutes. The AKW was measured on chest radiographs by a single reviewer.

Results: The AKW was significantly correlated with the HRV parameters, except for the LF/HF ratio. However, RMSSD and ApEn were not significantly related to the AKW in women. After dividing the participants into quartile groups, the AKW was significantly related to the SDNN, RMSSD, TP, VLF, LF, and HF. The HRV parameter values decreased in the higher AKW quartile groups, the HRV parameter values decreased. After adjusting for sex, drinking status, exercise habits, smoking status, waist circumference, and triglyceride, low-density lipoprotein cholesterol, fasting blood sugar, and glycated hemoglobin levels, the AKW showed significant negative associations with the HRV parameters, except for the LF/HF ratio.

Conclusion: The AKW is significantly associated with the HRV parameters of SDNN, RMSSD, ApEn, TP, VLF, LF, and HF.

Keywords: Heart Rate Variability; Aortic Knob Width; Cardiovascular Diseases; Thoracic Aorta

Received: May 29, 2018, Revised: September 18, 2018, Accepted: September 27, 2018

*Corresponding Author: Jee-Hye Han https://orcid.org/0000-0003-4002-3453

Tel: +82-2-970-8515, Fax: +82-2-970-8370, E-mail: hanjh1611@eulji.ac.kr 


\section{INTRODUCTION}

Heart rate variability (HRV) evaluates the activity of the autonomic nervous system (ANS) noninvasively. The normal activity of the ANS is determined by a balance between the sympathetic and parasympathetic activities. In the cardiovascular system, an imbalance in the ANS results in the fluctuation of the heart rate. Low HRV parameters were found to be associated with atherosclerotic plaque progression, congestive heart failure, myocardial infarction, arrhythmia, sudden cardiac death, diabetic neuropathy, hypertension, dyslipidemia, and older age. ${ }^{1-3)}$ Several studies showed that HRV may indicate a current illnesses or serve as a warning sign for various cardiac diseases, such as arrhythmia, myocardial infarction, coronary heart disease, and heart failure. ${ }^{1,2,45)}$

The aortic knob width (AKW) is an important parameter in chest radiography and may be an early indicator of cardiovascular diseases. It can be increased by changes in elasticity or by increased pressure flow in the aorta (e.g., in systemic hypertension and aortic dissection), and in the presence of the aortic arch calcification, atherosclerosis, and cardiovascular diseases. ${ }^{6-10)}$

No studies have investigated the correlation between the HRV and AKW, which is an important index of cardiovascular risk. This study aimed to investigate the relationship between these factors.

\section{METHODS}

\section{Subjects}

A total of 696 Korean adults aged 18-79 years who visited a health screening center for regular check-ups between January 2015 and July 2016 were considered for inclusion in this study. Subjects with a history of arrhythmia, atrial fibrillation, angina pectoris, myocardial infarction, cerebrovascular disease, major depression, thyroid disease, malignancy, and infectious diseases were excluded. Pregnant women and those who presented with incomplete questionnaires were also excluded. Out of the 696 subjects, 109 subjects were excluded, and the remaining 587 subjects were examined. All participants provided written informed consent prior to inclusion.

\section{Clinical Examination and Blood Assays}

Medical, social, and lifestyle histories, including drinking status, exercise habits, and smoking status, were collected using self-administered questionnaires. Based on the current drinking status, patients were divided into alcoholic drinkers and non-drinkers, while based on the current smoking status they were divided into smokers and nonsmokers. Only those who exercised regularly 3 or more times in a week were assigned to the exercise group.

The height and weight of participants were measured using an automatic digital stadiometer (GM-1000; NEO GM TEC, Incheon, Korea). Body mass index was calculated as body weight divided by height squared $\left(\mathrm{kg} / \mathrm{m}^{2}\right)$. Waist circumference was measured at the midpoint between the rib cage's lower border and the iliac crest. Blood pressure

Table 1. Characteristics of the study participants

\begin{tabular}{|c|c|c|c|c|}
\hline Characteristic & Total $(\mathrm{N}=587)$ & Men $(\mathrm{N}=351)$ & Women $(\mathrm{N}=236)$ & P-value \\
\hline Age $(y)$ & $46.85 \pm 12.89$ & $48.81 \pm 12.57$ & $43.93 \pm 12.83$ & $<0.001$ \\
\hline Aortic knob width (mm) & $32.08 \pm 5.54$ & $33.94 \pm 5.28$ & $29.30 \pm 4.71$ & $<0.001$ \\
\hline Body mass index $\left(\mathrm{kg} / \mathrm{m}^{2}\right)$ & $24.02 \pm 3.51$ & $25.01 \pm 3.25$ & $22.55 \pm 3.37$ & $<0.001$ \\
\hline Waist circumference $(\mathrm{cm})$ & $81.02 \pm 10.34$ & $85.47 \pm 8.66$ & $74.39 \pm 9.02$ & $<0.001$ \\
\hline Systolic BP (mm Hg) & $118.18 \pm 14.02$ & $122.26 \pm 12.28$ & $112.10 \pm 14.286$ & $<0.001$ \\
\hline Diastolic BP (mm Hg) & $70.99 \pm 10.09$ & $73.32 \pm 9.54$ & $67.52 \pm 9.93$ & $<0.001$ \\
\hline Total cholesterol (mg/dL) & $199.68 \pm 38.06$ & $202.19 \pm 38.04$ & $195.94 \pm 37.87$ & 0.051 \\
\hline Triglyceride (mg/dL) & $119.69 \pm 88.46$ & $142.43 \pm 100.664$ & $85.86 \pm 49.98$ & $<0.001$ \\
\hline High-density lipoprotein cholesterol (mg/dL) & $55.73 \pm 14.50$ & $51.11 \pm 11.97$ & $62.60 \pm 15.23$ & $<0.001$ \\
\hline Low-density lipoprotein cholesterol (mg/dL) & $120.63 \pm 33.90$ & $124.40 \pm 33.86$ & $115.01 \pm 33.23$ & 0.001 \\
\hline Fasting blood sugar (mg/dL) & $92.95 \pm 20.38$ & $95.69 \pm 23.67$ & $88.88 \pm 13.17$ & $<0.001$ \\
\hline Glycated hemoglobin levels (\%) & $5.56 \pm 0.70$ & $5.63 \pm 0.75$ & $5.45 \pm 0.60$ & 0.002 \\
\hline Mean heart rate (ms) & $70.38 \pm 9.85$ & $69.34 \pm 9.41$ & $71.93 \pm 10.30$ & 0.002 \\
\hline SD of all normal-to-normal intervals (ms) & $32.53 \pm 13.81$ & $32.75 \pm 14.38$ & $32.22 \pm 12.94$ & 0.649 \\
\hline Root mean square of successive differences (ms) & $23.44 \pm 12.46$ & $22.92 \pm 12.96$ & $24.21 \pm 11.67$ & 0.221 \\
\hline Approximate entropy & $0.97 \pm 0.11$ & $0.97 \pm 0.11$ & $0.96 \pm 0.11$ & 0.870 \\
\hline $\mathrm{TP}^{*}\left(\mathrm{~ms}^{2}\right)$ & $6.40 \pm 0.93$ & $6.41 \pm 0.96$ & $6.40 \pm 0.89$ & 0.896 \\
\hline Very LF $\left(\mathrm{ms}^{2}\right)$ & $5.54 \pm 1.09$ & $5.54 \pm 1.11$ & $5.55 \pm 1.07$ & 0.947 \\
\hline $\mathrm{LF}\left(\mathrm{ms}^{2}\right)$ & $5.03 \pm 1.09$ & $5.08 \pm 1.13$ & $4.95 \pm 1.03$ & 0.167 \\
\hline $\mathrm{HF}\left(\mathrm{ms}^{2}\right)$ & $4.62 \pm 1.16$ & $4.55 \pm 1.21$ & $4.73 \pm 1.07$ & 0.056 \\
\hline $\mathrm{LF} / \mathrm{HF}$ ratio & $2.67 \pm 4.13$ & $2.90 \pm 3.97$ & $2.33 \pm 4.34$ & 0.102 \\
\hline
\end{tabular}

Values are presented as mean \pm SD. All data were analyzed by independent t-test. P-values refer to comparisons between men and women.

BP, blood pressure; SD, standard deviation; TP, total power; LF, low-frequency; HF, high-frequency.

${ }^{*}$ Short-term estimate of the TP of power spectral density. 
(BP) was measured using an automated sphygmomanometer (FT500R; Jawon Medical, Kyungsan, Korea) after 5 minutes of stabilization.

Blood samples were collected the following morning after fasting for at least 8 hours. The laboratory tests included assessment of the total cholesterol, triglyceride, high-density lipoprotein cholesterol (HDL-C), low-density lipoprotein cholesterol (LDL-C), fasting blood sugar (FBS), and glycated hemoglobin ( $\mathrm{HbAlc}$ ) levels.

\section{Measurement of the Aortic Knob Width}

The aortic knob is best visualized on frontal chest radiographs. The AKW was measured along the horizontal line from the point of the trachea's lateral edge to the aortic knob's left lateral wall. ${ }^{7,11)}$ To minimize possible errors, all measurements were performed by one reviewer.

\section{Heart Rate Variability Measurement}

For accurate measurement, the following conditions were maintained: (1) a room temperature of $23^{\circ} \mathrm{C}-25^{\circ} \mathrm{C}$ and noise was avoided; (2) participants were asked to avoid consuming caffeine and smoking 2 hours before the examination; and (3) participants were asked to relax and not move or speak during the examination.

The HRV parameters were measured using a HRV analyzer (SA3000P; Medicore Co. Ltd., Seoul, Korea) for 5 minutes. The HRV provides significant information on the regulatory function and balance status of the ANS.

All HRV parameters were calculated on 'normal-to-normal' (N-N) inter-beat intervals ( $\mathrm{N}-\mathrm{N}$ intervals) caused by normal heart contrac- tions paced by the sinus node depolarization. Assessed parameters were measured by the time domain and frequency domain. ${ }^{2)}$ The time domain parameters used in this study included the standard deviation of the N-N interval (SDNN), root mean square of successive differences (RMSSD), and approximate entropy (ApEn). The SDNN is the square root of the variance and is a marker of total power (TP) in HRV. ${ }^{1)}$ Furthermore, it reflects both parasympathetic and sympathetic activities. The RMSSD is the square root of the mean squared differences of successive $\mathrm{N}-\mathrm{N}$ intervals. This parameter estimates high-frequency variations in the heart rate in short-term N-N recordings and reflects an estimate of parasympathetic activities. The ApEn is the quantification of the regularity and complexity of the HRV; the higher the ApEn, the higher the tachogram's complexity.

The frequency domain parameters used in this study included the TP (0-0.4 Hz), very low frequency (VLF, 0-0.4 Hz), low frequency (LF, $0.04-0.15 \mathrm{~Hz}$ ), high frequency (HF, 0.15-0.4 Hz), and LF/HF ratio. The TP mainly shows the level of both parasympathetic and sympathetic activities; a decreased TP indicates that the individual is under chronic stress or has some underlying disease. TP is strongly associated with the risks of sudden cardiac death and all-cause death after myocardial infarction. VLF is an additional indicator of sympathetic function. LF reflects sympathetic and parasympathetic activities in short-term measurements and sympathetic activities in long-term measurements. HF reflects the parasympathetic or vagal activity of the ANS; a reduced HF is related to aging, electrical instability of the heart, and chronic stress. The LF/HF ratio is an index that quantifies the overall balance between the sympathetic and parasympathetic nervous sys-

Table 2. Relationships between aortic knob width and investigated variables

\begin{tabular}{|c|c|c|c|c|c|c|}
\hline \multirow{2}{*}{ Variable } & \multicolumn{2}{|c|}{ Total } & \multicolumn{2}{|c|}{ Men } & \multicolumn{2}{|c|}{ Women } \\
\hline & $\mathrm{R}$ & P-value & $\mathrm{R}$ & P-value & $\mathrm{R}$ & P-value \\
\hline Age (y) & 0.583 & $<0.001$ & 0.5110 & $<0.001$ & 0.661 & $<0.001$ \\
\hline Body mass index $\left(\mathrm{kg} / \mathrm{m}^{2}\right)$ & 0.406 & $<0.001$ & 0.193 & $<0.001$ & 0.498 & $<0.001$ \\
\hline Waist circumference (cm) & 0.464 & $<0.001$ & 0.227 & $<0.001$ & 0.474 & $<0.001$ \\
\hline Systolic BP (mm Hg) & 0.424 & $<0.001$ & 0.206 & $<0.001$ & 0.506 & $<0.001$ \\
\hline Diastolic BP (mm Hg) & 0.349 & $<0.001$ & 0.192 & $<0.001$ & 0.388 & $<0.001$ \\
\hline Total cholesterol (mg/dL) & 0.083 & $<0.001$ & -0.061 & 0.255 & 0.249 & $<0.001$ \\
\hline Triglyceride (mg/dL) & 0.248 & $<0.001$ & 0.093 & 0.082 & 0.308 & $<0.001$ \\
\hline High-density lipoprotein cholesterol (mg/dL) & -0.265 & $<0.001$ & -0.064 & 0.229 & -0.212 & 0.001 \\
\hline Low-density lipoprotein cholesterol (mg/dL) & 0.116 & $<0.001$ & -0.096 & 0.072 & 0.346 & $<0.001$ \\
\hline Fasting blood sugar (mg/dL) & 0.275 & $<0.001$ & 0.206 & $<0.001$ & 0.322 & $<0.001$ \\
\hline Glycated hemoglobin levels (\%) & 0.296 & $<0.001$ & 0.253 & $<0.001$ & 0.302 & $<0.001$ \\
\hline Mean heart rate $(\mathrm{ms})$ & -0.145 & $<0.001$ & -0.040 & 0.459 & -0.197 & 0.002 \\
\hline Standard deviation of all normal-to-normal intervals (ms) & -0.207 & $<0.001$ & -0.223 & $<0.001$ & -0.257 & $<0.001$ \\
\hline Root mean square of successive differences (ms) & -0.143 & $<0.001$ & -0.144 & 0.007 & -0.116 & 0.075 \\
\hline Approximate entropy & -0.113 & $<0.001$ & -0.148 & 0.006 & -0.091 & 0.162 \\
\hline $\mathrm{TP}^{*}\left(\mathrm{~ms}^{2}\right)$ & -0.244 & $<0.001$ & -0.290 & $<0.001$ & -0.234 & $<0.001$ \\
\hline Very LF $\left(\mathrm{ms}^{2}\right)$ & -0.159 & $<0.001$ & -0.186 & $<0.001$ & -0.152 & $<0.001$ \\
\hline $\operatorname{LF}\left(\mathrm{ms}^{2}\right)$ & -0.269 & $<0.001$ & -0.362 & $<0.001$ & -0.246 & $<0.001$ \\
\hline $\mathrm{HF}\left(\mathrm{ms}^{2}\right)$ & -0.255 & $<0.001$ & -0.269 & $<0.001$ & -0.199 & $<0.001$ \\
\hline LF/HF ratio & 0.020 & 0.629 & -0.009 & 0.865 & -0.008 & 0.905 \\
\hline
\end{tabular}

All correlation coefficients were obtained by Pearson's correlation analysis. BP, blood pressure; TP, total power; LF, low-frequency; HF, high-frequency.

*Short-term estimate of the TP of power spectral density. 
tems. A high $\mathrm{LF} / \mathrm{HF}$ ratio indicates an increased sympathetic activity or reduced parasympathetic activity. All frequency domain parameters were transformed into natural logarithm.

\section{Statistical Analysis}

All continuous variables were analyzed using the independent t-test. Pearson's correlation analysis was conducted to determine the association between the HRV parameters and the AKW. To evaluate the trends of each HRV parameter according to the AKW quartiles, the Pvalue for trend was obtained using the analysis of covariance, after adjusting for age, drinking status, smoking status, and exercise habits. Multiple regression analysis was used to investigate the relationship between each of the HRV parameters and the AKW. All analyses were two-tailed, and P-values $<0.05$ were considered statistically significant. All statistical analyses were performed using PASW SPSS for Windows ver. 18.0 (SPSS Inc., Chicago, IL, USA).

\section{RESULTS}

The study included a total of 587 participants: 351 men and 236 women. The mean patient age was $46.85 \pm 12.89$ years. The mean AKW was $32.08 \pm 5.54 \mathrm{~mm}$ in the general study population, $33.94 \pm 5.28 \mathrm{~mm}$ in men, and $29.30 \pm 4.71 \mathrm{~mm}$ in women. No significant differences were found between men and women in any HRV parameter, except for the mean heart rate (Table 1).
The AKW was significantly negatively associated with all HRV parameters in all participants, except for the LF/HF ratio. In men, the AKW was significantly negatively associated with SDNN (ms), RMSSD (ms), ApEn, TP, VLF, LF, and HF. In women, the AKW was significantly negative associated with SDNN (ms), TP, VLF, LF, and HF (Table 2).

After adjusting for drinking status, exercise habits, smoking status, waist circumference, and triglyceride, LDL-C, FBS, and HbAlc levels, all HRV parameters decreased as the AKW quartile increased (Table 3).

The AKW was significantly associated with the SDNN $\left(\mathrm{R}^{2}=0.310\right.$, $\mathrm{P}<0.001)$, RMSSD $\left(\mathrm{R}^{2}=0.306, \mathrm{P}=0.048\right)$, ApEn $\left(\mathrm{R}^{2}=0.306, \mathrm{P}=0.001\right)$, TP $\left(\mathrm{R}^{2}=0.320, \mathrm{P}<0.001\right), \operatorname{VLF}\left(\mathrm{R}^{2}=0.303, \mathrm{P}=0.004\right), \mathrm{LF}\left(\mathrm{R}^{2}=0.337, \mathrm{P}<0.001\right)$, and $\mathrm{HF}\left(\mathrm{R}^{2}=0.315, \mathrm{P}<0.001\right)$ after adjusting for sex, drinking status, exercise habits, smoking status, waist circumference, and triglyceride, LDL-C, FBS, and HbAlc levels (Table 4).

\section{DISCUSSION}

The HRV parameters were not significantly different between men and women, except for the mean heart rate. As the quartile of the AKW increased, the HRV parameters decreased. This negative correlation was enhanced in the multiple regression analysis after adjusting for significant variables.

In contrast, a previous study has shown that the RMSSD and HF, which reflect parasympathetic activities, were higher in women than in men. ${ }^{12)}$ Moreover, the quartile of each AKW was higher in men than

Table 3. Trends of each heart rate variability parameter according to quartiles of AKW

\begin{tabular}{|c|c|c|c|c|c|c|}
\hline & \multirow{2}{*}{ Variable } & \multicolumn{4}{|c|}{ Quartiles of AKW } & \multirow{2}{*}{$\begin{array}{l}\text { P-value } \\
\text { for trend }\end{array}$} \\
\hline & & Q1 & Q2 & Q3 & Q4 & \\
\hline \multirow[t]{7}{*}{ Total } & Range of AKW (mm) & $20.74-27.88$ & $27.89-31.72$ & $31.73-35.15$ & $35.16-75.00$ & \\
\hline & SDNN (ms) & $37.64 \pm 13.99$ & $32.95 \pm 12.31$ & $31.60 \pm 13.97$ & $27.89 \pm 13.14$ & $<0.001$ \\
\hline & RMSSD (ms) & $26.15 \pm 14.18$ & $23.52 \pm 11.16$ & $22.77 \pm 10.10$ & $21.27 \pm 23.80$ & 0.001 \\
\hline & $\mathrm{TP}\left(\mathrm{ms}^{2}\right)$ & $6.77 \pm 0.75$ & $6.49 \pm 0.88$ & $6.30 \pm 0.99$ & $6.05 \pm 0.94$ & $<0.001$ \\
\hline & $\operatorname{VLF}\left(\mathrm{ms}^{2}\right)$ & $5.86 \pm 0.99$ & $5.57 \pm 1.08$ & $5.48 \pm 1.16$ & $5.26 \pm 1.05$ & $<0.001$ \\
\hline & $\mathrm{LF}\left(\mathrm{ms}^{2}\right)$ & $5.46 \pm 0.93$ & $5.21 \pm 1.00$ & $4.85 \pm 1.11$ & $4.59 \pm 1.11$ & $<0.001$ \\
\hline & $\mathrm{HF}\left(\mathrm{ms}^{2}\right)$ & $4.99 \pm 1.03$ & $4.74 \pm 1.05$ & $4.51 \pm 1.14$ & $4.23 \pm 1.27$ & $<0.001$ \\
\hline \multirow[t]{7}{*}{ Men } & Range of AKW (mm) & $22.02-30.45$ & $30.46-33.50$ & $33.51-36.69$ & $36.70-75.00$ & \\
\hline & SDNN (ms) & $37.74 \pm 14.11$ & $32.31 \pm 12.86$ & $32.32 \pm 14.52$ & $28.61 \pm 14.72$ & $<0.001$ \\
\hline & RMSSD (ms) & $25.56 \pm 14.76$ & $22.50 \pm 11.33$ & $22.78 \pm 11.11$ & $20.86 \pm 14.00$ & 0.025 \\
\hline & $\mathrm{TP}\left(\mathrm{ms}^{2}\right)$ & $6.84 \pm 0.74$ & $6.43 \pm 0.95$ & $6.33 \pm 0.99$ & $6.02 \pm 0.97$ & $<0.001$ \\
\hline & $\operatorname{VLF}\left(\mathrm{ms}^{2}\right)$ & $5.90 \pm 0.99$ & $5.54 \pm 1.12$ & $5.49 \pm 1.21$ & $5.24 \pm 1.00$ & $<0.001$ \\
\hline & $\mathrm{LF}\left(m \mathrm{~s}^{2}\right)$ & $5.64 \pm 0.92$ & $5.18 \pm 1.06$ & $4.92 \pm 1.07$ & $4.56 \pm 1.21$ & $<0.001$ \\
\hline & $\mathrm{HF}\left(\mathrm{ms}^{2}\right)$ & $5.00 \pm 1.02$ & $4.57 \pm 1.13$ & $4.50 \pm 1.13$ & $4.12 \pm 1.38$ & $<0.001$ \\
\hline \multirow[t]{7}{*}{ Women } & Range of AKW (mm) & $20.74-25.93$ & 25.94-28.50 & $28.51-32.00$ & $32.10-47.33$ & \\
\hline & SDNN (ms) & $37.42 \pm 13.93$ & $33.95 \pm 11.71$ & $30.55 \pm 13.19$ & $26.83 \pm 10.37$ & $<0.001$ \\
\hline & RMSSD (ms) & $26.99 \pm 13.38$ & $25.13 \pm 10.81$ & $22.75 \pm 10.92$ & $21.89 \pm 10.84$ & 0.009 \\
\hline & $\mathrm{TP}\left(\mathrm{ms}^{2}\right)$ & $6.66 \pm 0.76$ & $6.58 \pm 0.76$ & $6.27 \pm 0.99$ & $6.08 \pm 0.91$ & $<0.001$ \\
\hline & $\operatorname{VLF}\left(\mathrm{ms}^{2}\right)$ & $5.81 \pm 0.99$ & $5.6 \pm 1.03$ & $5.46 \pm 1.07$ & $5.29 \pm 1.13$ & 0.006 \\
\hline & $\mathrm{LF}\left(\mathrm{ms}^{2}\right)$ & $5.20 \pm 0.89$ & $5.25 \pm 0.92$ & $4.73 \pm 1.18$ & $4.62 \pm 0.96$ & $<0.001$ \\
\hline & $\mathrm{HF}\left(\mathrm{ms}^{2}\right)$ & $4.99 \pm 1.03$ & $5.00 \pm 0.87$ & $4.53 \pm 1.16$ & $4.40 \pm 1.09$ & $<0.001$ \\
\hline
\end{tabular}

Values are presented as mean \pm standard deviation. Analyzed by analysis of variance after adjusting for age, drinking status, exercise habits, and smoking status. P-value for trend was obtained by using analysis of covariance after adjusting for age, drinking status, exercise habits, and smoking status.

AKW, aortic knob width; SDNN, standard deviation of all normal-to-normal intervals, RMSSD, root mean square of successive differences; TP, short-term estimate of the total power of power spectral density; VLF, very low-frequency; LF, low-frequency; HF, high-frequency. 
Table 4. Multiple linear regression analysis of the association between the aortic knob width with the heart rate variability parameters

\begin{tabular}{|c|c|c|c|c|}
\hline Variable & $\beta \pm$ standard error & $\mathrm{R}^{2}$ & Adjusted $\mathrm{R}^{2}$ & P-value \\
\hline Standard deviation of all normal-to-normal intervals (ms) & $-0.054 \pm 0.014$ & 0.310 & 0.299 & $<0.001$ \\
\hline Root mean square of successive differences (ms) & $-0.031 \pm 0.16$ & 0.306 & 0.301 & 0.048 \\
\hline Approximate entropy & $-5.815 \pm 1.805$ & 0.306 & 0.295 & 0.001 \\
\hline $\mathrm{TP}^{*}\left(m \mathrm{~s}^{2}\right)$ & $-1.011 \pm 0.213$ & 0.320 & 0.309 & $<0.001$ \\
\hline Very LF $\left(\mathrm{ms}^{2}\right)$ & $-0.520 \pm 0.181$ & 0.303 & 0.292 & 0.004 \\
\hline $\mathrm{LF}\left(\mathrm{ms}^{2}\right)$ & $-1.109 \pm 0.180$ & 0.337 & 0.327 & $<0.001$ \\
\hline $\mathrm{HF}\left(\mathrm{ms}^{2}\right)$ & $-0.736 \pm 0.172$ & 0.315 & 0.304 & $<0.001$ \\
\hline
\end{tabular}

Multiple regression analysis was performed after adjusting for sex, drinking status, exercise habits, smoking status, waist circumference, and triglyceride, low-density lipoprotein cholesterol, fasting blood sugar, and glycated hemoglobin levels.

TP, total power; LF, low-frequency; HF, high-frequency.

*Short-term estimate of the total power of power spectral density.

in women in this study; this result is consistent with that of a previous study. ${ }^{13)}$

Previous studies have indicated that HRV and the AKW are associated with the increased risk for cardiovascular diseases. ${ }^{1,4,7,8,14,15)}$ However, the relationship between the AKW and HRV has not been yet elucidated. Except for the RMSSD and ApEn in the women, the AKW was highly correlated with the SDNN, RMSSD, ApEn, TP, VLF, LF, and HF. This relationship between AKW and HRV may be explained by several factors. First, both the HRV and AKW were found to be associated with coronary instability. ${ }^{1,12)}$ Second, increased BP in the aorta induces widening of the aortic knob. A low SDNN at a young age indicates an increased risk of hypertension. ${ }^{1,16)}$ Third, common factors, including age, hypertension, metabolic syndrome, atherosclerosis, and diabetes mellitus, were found to be associated with the AKW widening and decreased HRV ${ }^{1,16-20)}$ Finally, unstable hemodynamics causing aortic arch calcification is presumed to be related to an imbalance in the ANS activity. ${ }^{9,10,21,22)}$

The HRV parameters were divided into two categories: time domain and frequency domain. The parameters of the time domain were the SDNN, RMSSD, and ApEn, while the parameters of the frequency domain were the TP, VLF, LF, HF, and LF/HF ratio. ${ }^{1,4,14)}$ The data obtained from short-term (5 minutes) recordings should be processed with frequency domain methods, whereas time domain analyses should be performed to analyze 24-hour, long-term recordings. ${ }^{1)}$ This study used the 5-minute measurement method, and the frequency domain parameters were matched well regardless of sex. Therefore, we can assume that the frequency domain parameters matched better in the correlation analysis between the AKW and HRV.

This study has several limitations. The long-term analysis (24-hour measurement) is more accurate than the short-term analysis (5-minute analysis). However, the short-term analysis is less expensive, saves time, and is convenient for the participants. A large number of subjects should be investigated in future studies to clarify the relationship between the AKW and HRV parameters.

Despite these limitations, HRV can be a useful factor in evaluating the change in AKW associated with cardiovascular disease. If an individual has a significantly higher AKW than the average age group, we can assume that such an individual has low SDNN and TP (represent- ing HRV). The result was obtained using chest radiography; thus, HRV may be used clinically as an indicator of possible cardiovascular diseases. Further research is needed to determine how HRV can be used as a clinical factor to evaluate cardiac risks, and how to apply the relationship between the AKW and HRV. In addition, it is necessary to investigate the relationship between the AKW and HRV in metabolic syndrome and the change in this relationship according to age and sex.

In conclusion, the HRV parameters were significantly associated with the AKW in the Korean population of this study.

\section{CONFLICT OF INTEREST}

No potential conflict of interest relevant to this article was reported.

\section{ORCID}

Jee-Hye Han: https://orcid.org/0000-0003-4002-3453

Jung-Hwan Kim: https://orcid.org/0000-0001-8984-2914

\section{REFERENCES}

1. Xhyheri B, Manfrini O, Mazzolini M, Pizzi C, Bugiardini R. Heart rate variability today. Prog Cardiovasc Dis 2012;55:321-31.

2. Malik M, Camm AJ. Heart rate variability. Clin Cardiol 1990;13:570-6.

3. Task Force of the European Society of Cardiology and the North American Society of Pacing and Electrophysiology. Heart rate variability: standards of measurement, physiological interpretation, and clinical use. Eur Heart J 1996;17:354-81.

4. ChuDuc H, NguyenPhan K, NguyenViet D. A review of heart rate variability and its applications. APCBEE Procedia 2013;7:80-5.

5. Bilchick KC, Berger RD. Heart rate variability. J Cardiovasc Electrophysiol 2006;17:691-4.

6. Rayner BL, Goodman H, Opie LH. The chest radiograph: a useful investigation in the evaluation of hypertensive patients. Am J Hypertens 2004;17:507-10.

7. Yun KH, Jeong MH, Oh SK, Park EM, Kim YK, Rhee SJ, et al. Clinical significance of aortic knob width and calcification in unstable angina. Circ J 2006;70:1280-3. 
8. Anyanwu GE, Anibeze CI, Akpuaka FC. Transverse aortic arch diameters and relationship with heart size of Nigerians within the South East. Biomed Res 2007;18:115-8.

9. Iijima K, Hashimoto H, Hashimoto M, Son BK, Ota H, Ogawa S, et al. Aortic arch calcification detectable on chest X-ray is a strong independent predictor of cardiovascular events beyond traditional risk factors. Atherosclerosis 2010;210:137-44.

10. Craiem D, Alsac JM, Casciaro ME, El Batti S, Mousseaux E, Sirieix ME, et al. Association between thoracic aorta calcium and thoracic aorta geometry in a cohort of asymptomatic participants at increased cardiovascular risk. Rev Esp Cardiol (Engl Ed) 2016;69:827-35.

11. Kurisu S, Higaki T, Sumimoto Y, Ikenaga H, Watanabe N, Ishibashi K, et al. Aortic knob width reflects left ventricular diastolic function assessed by gated myocardial perfusion single photon emission computed tomography in patients with normal myocardial perfusion. Ann Nucl Med 2017;31:245-9.

12. Antelmi I, de Paula RS, Shinzato AR, Peres CA, Mansur AJ, Grupi CJ. Influence of age, gender, body mass index, and functional capacity on heart rate variability in a cohort of subjects without heart disease. Am J Cardiol 2004;93:381-5.

13. Ray A, Mandal D, Kundu P, Manna S, Mandal S. Aortic knob diameter in chest X-ray and its relation with age, heart diameter and transverse diameter of thorax in a population of Bankura District of West Bengal, India: a cross sectional study. J Evol Med Dent Sci 2014;3:8595-600.

14. Rajendra Acharya U, Paul Joseph K, Kannathal N, Lim CM, Suri JS. Heart rate variability: a review. Med Biol Eng Comput 2006;44:103151.

15. Erdem K, Ozturk S, Ayhan S, Bugra O, Bozoglan O, Tekelioglu UY, et al. Predictive value of aortic knob width for postoperative atrial fibrillation in coronary artery bypass surgery. Anadolu Kardiyol Derg 2014; 14:68-72.

16. Liao D, Sloan RP, Cascio WE, Folsom AR, Liese AD, Evans GW, et al. Multiple metabolic syndrome is associated with lower heart rate vari- ability: the atherosclerosis risk in communities study. Diabetes Care 1998;21:2116-22.

17. Erbel R, Aboyans V, Boileau C, Bossone E, Bartolomeo RD, Eggebrecht $\mathrm{H}$, et al. 2014 ESC guidelines on the diagnosis and treatment of aortic diseases: document covering acute and chronic aortic diseases of the thoracic and abdominal aorta of the adult: the Task Force for the Diagnosis and Treatment of Aortic Diseases of the European Society of Cardiology (ESC). Eur Heart J 2014;35:2873-926.

18. Hiratzka LF, Bakris GL, Beckman JA, Bersin RM, Carr VF, Casey DE Jr, et al. 2010 ACCF/AHA/AATS/ACR/ASA/SCA/SCAI/SIR/STS/SVM guidelines for the diagnosis and management of patients with thoracic aortic disease: a report of the American College of Cardiology Foundation/American Heart Association Task Force on practice guidelines, American Association for Thoracic Surgery, American College of Radiology, American Stroke Association, Society of Cardiovascular Anesthesiologists, Society for Cardiovascular Angiography and Interventions, Society of Interventional Radiology, Society of Thoracic Surgeons, and Society for Vascular Medicine. J Am Coll Cardiol 2010;55:e27-e129.

19. Collins JA, Munoz JV, Patel TR, Loukas M, Tubbs RS. The anatomy of the aging aorta. Clin Anat 2014;27:463-6.

20. Redheuil A, Yu WC, Mousseaux E, Harouni AA, Kachenoura N, Wu $\mathrm{CO}$, et al. Age-related changes in aortic arch geometry: relationship with proximal aortic function and left ventricular mass and remodeling. J Am Coll Cardiol 2011;58:1262-70.

21. Roach D, Sheldon A, Wilson W, Sheldon R. Temporally localized contributions to measures of large-scale heart rate variability. Am J Physiol 1998;274(5 Pt 2):H1465-71.

22. Yang TL, Huang CC, Huang SS, Chiu CC, Leu HB, Lin SJ. Aortic arch calcification associated with cardiovascular events and death among patients with acute coronary syndrome. Acta Cardiol Sin 2017;33:2419. 